\title{
Motivation-focused thinking: Buffering against stress-related physical symptoms and depressive symptomology
}

\author{
Jeremy M. Hamm ${ }^{\mathrm{a} *}$, Raymond P. Perry ${ }^{\mathrm{a}}$, Judith G. Chipperfield ${ }^{\mathrm{a}}$, Tara L. Stewart ${ }^{\mathrm{b}}$ and \\ Jutta Heckhausen $^{\text {c }}$ \\ ${ }^{a}$ Department of Psychology, University of Manitoba, Winnipeg, MB, Canada; ${ }^{b}$ Winnipeg Regional \\ Health Authority and the Centre for Healthcare Innovation, University of Manitoba, Winnipeg, \\ MB, Canada; ${ }^{c}$ Department of Psychology and Social Behavior, University of California, Irvine, \\ Irvine, CA, USA
}

(Received 29 January 2015; accepted 6 May 2015)

\begin{abstract}
Developmental transitions are experienced throughout the life course and necessitate adapting to consequential and unpredictable changes that can undermine health. Our six-month study $(n=239)$ explored whether selective secondary control striving (motivation-focused thinking) protects against the elevated levels of stress and depressive symptoms increasingly common to young adults navigating the challenging school-to-university transition. Path analyses supplemented with tests of moderated mediation revealed that, for young adults who face challenging obstacles to goal attainment, selective secondary control indirectly reduced long-term stress-related physical and depressive symptoms through selective primary control and previously unexamined measures of discrete emotions. Results advance the existing literature by demonstrating that (a) selective secondary control has health benefits for vulnerable young adults and (b) these benefits are largely a consequence of the process variables proposed in Heckhausen et al.'s (2010) theory.
\end{abstract}

Keywords: motivation; goal engagement; primary and secondary control; psychological and physical health

Whether one is a five-year old entering kindergarten or an 85-year old entering a personal care home, adjusting to change throughout the lifespan is often difficult. Notably, there may be few periods during the normal life course that necessitate adapting to more significant and unpredictable changes than developmental transitions (Heckhausen \& Schulz, 1995; Perry, 2003). Consider, for example, the consequential shift from high school to university. During this developmental transition, young adults must navigate a variety of novel changes and challenges, including increased pressures to excel, frequent failures, new living arrangements, unstable social networks, financial responsibilities and critical career choices (Perry, 1991, 2003). Thus, it may be unsurprising that the considerable changes experienced during this challenging transition have the capacity to overwhelm young adults and undermine psychological and physical health (Adlaf, Gliksman, Demers, \& Newton-Taylor, 2001; Hussain, Guppy, Robertson, \& Temple, 2013).

\footnotetext{
*Corresponding author. Email: umhamm8@myumanitoba.ca
} 
For instance, a recent American Psychological Association report suggests young adults experience stress levels that are among the highest of any age bracket and are the most likely to report their stress has increased over the past five years (American Psychological Association [APA], 2012). Elevated levels of stress are implicated in poor health and are commonly manifest in physical symptoms, such as fatigue, headaches and muscular tension (APA, 2012). Thus, although young adulthood is commonly conceived of as period of health, students navigating the school-to-university transition may experience physical health problems similar to or worse than the general population (Grace, 1997; Hussain et al., 2013).

More concerning, an American College Health Association survey of over 28,000 university students found that, within the last year, 45\% felt things were hopeless, $50 \%$ experienced immense anxiety and $85 \%$ were overwhelmed by their responsibilities (American College Health Association [ACHA], 2012). Further, 30\% of students reported being so depressed that they had difficulty functioning at least once during the past 12 months (ACHA, 2012). Of note, the fact that $11 \%$ of respondents were either diagnosed or treated for depression during the last year suggests that university students are approximately $57 \%$ more likely to be clinically depressed than adults in the general American population (ACHA, 2012; Kessler, Chiu, Demler, \& Walters, 2005). ${ }^{1}$

Thus, the elevated levels of stress and depression increasingly common in young adulthood underscore the need for further research on psychological factors that may promote well-being during the challenging transition from school to university. Preliminary evidence stemming from the motivational theory of lifespan development (Heckhausen, Wrosch, \& Schulz, 2010) points to the buffering influence of selective secondary control. Employing these motivation-focused strategies has been found to facilitate motivation, goal striving and goal attainment for individuals navigating difficult developmental transitions (Hamm et al., 2013; Poulin \& Heckhausen, 2007). However, researchers have yet to adequately explore whether the benefits of this protective form of motivation-focused thinking extend to pertinent health outcomes for young adults.

Consequently, the present study focused on the influence of selective secondary control striving on stress-related physical symptoms and depressive symptomology among young adults negotiating the challenging school-to-university transition. Because selective secondary control is posited to particularly advantage individuals who encounter barriers during goal pursuit (Heckhausen et al., 2010), we examined whether unprepared university students facing educational obstacles experienced the most pronounced benefits from this form of motivation-focused thinking. However, drawing on theory suggesting that selective secondary control exerts its influence indirectly (Heckhausen et al., 2010), we also explored whether selective secondary control's effects were mediated by pertinent psychological mechanisms. Thus, our study expanded on previous research by using moderated mediation and path analysis to simultaneously test two intertwined theoretical propositions proposed by Heckhausen et al. (2010): that the effects of selective secondary control should be indirect and primarily benefit those facing challenging obstacles.

\section{The protective effects of selective secondary control for young adults}

The motivational theory of lifespan development (Heckhausen \& Schulz, 1995; Heckhausen, Wrosch, \& Fleeson, 2001; Heckhausen et al., 2010) provided a theoretical 
basis for our examination of the effects of selective secondary control strategies on health among young adults. Heckhausen et al. (2010) posit that humans commonly employ selective primary and selective secondary control strategies during goal pursuit. Selective primary control involves strategies that target external behavioural resources in order to pursue goals (e.g. increasing time on task). Selective secondary control involves strategies that target internal cognitive and affective resources in order to sustain volitional goal commitment (e.g. downplaying conflicting goals).

Past research suggests that simultaneously employing selective primary and selective secondary control strategies (i.e. goal engagement) has important consequences for psychological and physical health. Higher levels of goal engagement are related to increased positive affect, higher life satisfaction, increased life purpose, fewer depressive symptoms, decreased chronic conditions, better functional status and reduced diurnal cortisol secretion for adults across the lifespan (Haase, Heckhausen, \& Köller, 2008; Haase, Heckhausen, \& Silbereisen, 2012; Mackay, Charles, Kemp, \& Heckhausen, 2011; Wrosch \& Schulz, 2008; Wrosch, Schulz, Miller, Lupien, \& Dunne, 2007).

Several studies have attempted to tease apart the influence of these two goal engagement strategies by documenting the independent health effects of selective primary and selective secondary control striving. For instance, over a decade of research suggests that employing selective primary control strategies promotes long-term psychological (e.g. increased positive affect) and physical health (e.g. reduced physical symptoms; Chipperfield \& Perry, 2006; Chipperfield, Perry, \& Menec, 1999; Hall, Chipperfield, Heckhausen, \& Perry, 2010; Haynes, Heckhausen, Chipperfield, Perry, \& Newall, 2009; Windsor, 2009). Research examining the unique influence of selective secondary control is limited but in line with the broader goal engagement literature: Employing these motivation-focused strategies is related to improved positive affect in older adults experiencing macular degeneration (Wahl, Becker, Burmedi, \& Schilling, 2004).

Despite this impressive body of evidence, several noteworthy gaps remain in the literature. First, researchers have neglected to explore the effects of selective secondary control on consequential health outcomes for young adults negotiating the challenging developmental transition from high school to university. ${ }^{2}$ Considering that theory and research suggest this form of motivation-focused thinking is of greatest benefit when facing challenging circumstances (Hamm et al., 2013; Heckhausen et al., 2010; Poulin \& Heckhausen, 2007), selective secondary control may buffer against the elevated levels of stress and depression increasingly common to young adults in the midst of this transition (ACHA, 2012; APA, 2012).

Although the benefits of this form of motivation-focused thinking are implied in the aforementioned body of evidence relating goal engagement (i.e. simultaneous employment of selective secondary and selective primary control strategies) to health, the majority of this research was conducted on older adults (e.g. Mackay et al., 2011; Wrosch \& Schulz, 2008; Wrosch et al., 2007). Interestingly, however, both younger and older adults are forced to navigate challenging developmental transitions that have the capacity to undermine motivation and goal striving (see Hamm, Chipperfield, Perry, Heckhausen, \& Mackenzie, 2014; Perry, 2003). Consequently, theory suggests that selective secondary control striving should confer health benefits on young and old adults alike given that this form of motivation-focused thinking is posited to be most adaptive when facing difficult obstacles, such as those frequently encountered during developmental transitions (Heckhausen et al., 2010). Hence, empirical evidence is 
needed to establish whether selective secondary control striving sustains critical health outcomes for young adults in transition in line with the benefits observed for older adults.

A second limitation in the literature is that researchers have yet to adequately explore the theoretical proposition that selective secondary control should especially advantage high-risk individuals prone to initial failure during goal pursuit (Heckhausen et al., 2010). Thus far, only Poulin and Heckhausen's (2007) study has examined this issue. However, their focus was on the buffering effect of selective secondary control for adolescents experiencing extraordinary, abrupt and external stressors in their personal lives (i.e. family member death or parental divorce). As described earlier, selective secondary control is theorized to confer the greatest benefit on individuals encountering barriers during goal pursuit (Heckhausen et al., 2010). Although extraordinary, abrupt stressors represent one type of barrier, more stable, everyday obstacles may represent an equally challenging barrier to attaining consequential long-term goals. Thus, it follows that this form of motivation-focused thinking may particularly advantage young adults facing stable educational obstacles during the challenging school-to-university transition.

Perhaps the most common, stable educational obstacle faced by young adults aspiring to earn university degrees and ultimately secure gainful employment is beginning post-secondary education with a low high school grade (HSG). A comprehensive metaanalysis conducted by Richardson, Abraham, and Bond (2012) revealed that HSG is the strongest traditional correlate of university grade point averages (GPAs), predicting post-secondary achievement as well or better than SAT or ACT scores. Moreover, in a job market inundated with college graduates, distinguishing one's self will require more than simply completing a series of degree requirements and may increasingly depend on university GPA. In fact, GPA predicts future education level, occupational status and income, highlighting the importance of post-secondary achievement in realising coveted long-term goals (Strenze, 2007).

Thus, poor academic performance in high school can signify a lack of preparation and may exacerbate the already considerable challenges inherent in the school-to -university transition. As a result, employing selective secondary control strategies as a means of coping with the academic and career impediments posed by poor high school achievement represents an adaptive response to a significant threat to one's goal pursuit. ${ }^{3}$ Such a strategic approach may not only sustain motivation and goal striving for young adults with low HSGs who are unprepared for university, it may also protect them from experiencing otherwise elevated levels of stress-related physical symptoms and depression. However, further research is needed to examine this issue.

A final limitation is that, with few exceptions, previous research has largely overlooked intervening mechanisms that explain why selective secondary control is beneficial. Notably, in line with theoretical propositions stemming from Heckhausen et al. (2010), a study by Hamm et al. (2013) demonstrated that this form of motivationfocused thinking is a strong predictor of longitudinal selective primary control striving among young adults. Previous research also suggests that higher levels of selective primary control striving are related to reduced stress and depression (Haynes et al., 2009; Heckhausen et al., 2001; Schilling et al., 2013). Consequently, selective primary control may represent one mechanism for selective secondary control striving to sustain health among young adults. 
Interestingly, however, the influence of selective primary control on critical health outcomes, including stress and depression, may be further mediated by emotional wellbeing. Past studies suggest that selective primary control striving is associated with increased positive and decreased negative affect (e.g. Haase et al., 2012; Haynes et al., 2009; Windsor, 2009). In turn, an extensive literature demonstrates that emotional wellbeing is strongly related to improved psychological (e.g. depressive symptoms) and physical (e.g. physical symptoms) health outcomes for adults across the lifespan (e.g. Chipperfield, Perry, \& Weiner, 2003; Ostir, Markides, Black, \& Goodwin, 2000; Pekrun et al., 2004; see Lyubomirsky, King, \& Diener, 2005 for a review of 225 studies).

Collectively, this research implies that selective secondary control may indirectly promote health via a sequence of theoretically driven psychological mechanisms involving selective primary control and emotional well-being. However, additional research is required to determine (a) whether motivation-focused thinking indirectly predicts discrete emotions via selective primary control, and (b) whether selective primary control and discrete emotions transmit the protective influence of selective secondary control to stress-related physical symptoms and depression.

Consequently, our study was designed to expand on previous research and address the aforementioned gaps in the literature by examining whether selective secondary control striving buffered against long-term stress-related physical symptoms and depressive symptomology during the challenging shift from high school to university. We were particularly interested in determining whether selective secondary control was most beneficial for young adults facing challenging educational obstacles (i.e. low HSGs). However, we were equally concerned with testing whether selective primary control and discrete emotions provided the mechanisms through which selective secondary control benefited health. Based on Heckhausen et al. (2010) and the preceding logic, we expected that, for students with low HSGs, (a) selective secondary control would promote selective primary control, (b) selective primary control would foster positive emotion and suppress negative emotion and (c) selective primary control and the emotions would predict stress-related physical and depressive symptoms (see Figure 1).

A supplemental objective was to examine whether selective secondary control striving facilitated long-term goal attainment for young adults with low HSGs given that
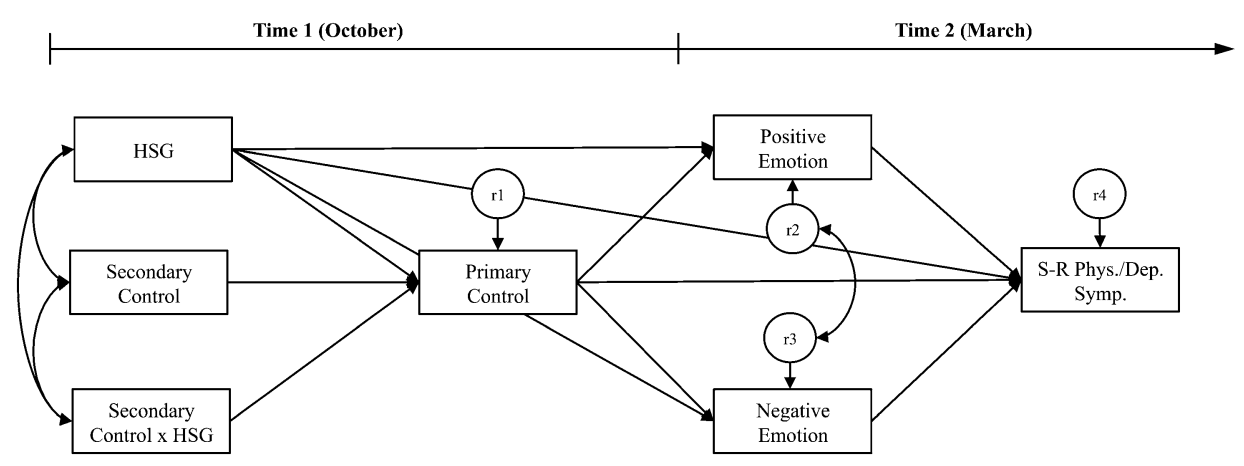

Figure 1. Structural model displaying all specified paths for the hypothesised sequence predicting stress-related physical symptoms (S-R phys. symp.) and depressive symptoms (dep. symp.). Notes: All effects were adjusted for age and gender. $\mathrm{HSG}=$ high school grade, $r=$ residual. 
these individuals are at increased risk of academic failure (Richardson et al., 2012). Based on past research suggesting these motivation-focused strategies foster goal attainment for individuals facing challenging circumstances (Hamm et al., 2013; Poulin \& Heckhausen, 2007), we expected selective secondary control to indirectly promote longterm academic performance for students with low HSGs via the hypothesised sequence of psychological mechanisms depicted in Figure 1.

\section{Method}

\section{Participants and procedures}

We drew our study sample from a comprehensive database that contains psychosocial data for over 20 separate cohorts of introductory psychology students at a research-intensive university in Western Canada $(n>20,000 ; 1992-2014)$. We used the 2007-2008 cohort for our analyses because data were collected on all variables of interest. Three phases were used to collect data on students who were recruited via a departmental participant pool system and participated in exchange for experimental credit. Participants completed a Time 1 (October) questionnaire early in the year and returned five months later to complete a similar Time 2 (March) questionnaire. Time 3 (April) involved acquiring consenting participants' academic performance data from their course instructors after the second semester concluded. The majority of participants in this sample $(n=239)$ were 17-20-year-old (80\%) females (72\%) who were native English speakers $(77 \%)$ enrolled full time $(91 \%)$ in their first year of university $(69 \%)$.

\section{Covariates}

Age (Time 1)

Participants reported their age using a 10-point scale $(1=17-18,10=$ older than 45$)$. Because approximately half the students indicated they were between the ages of 17 and 18 , and since the first year of university represents a challenging developmental transition for young adults (Perry, 1991, 2003), age was dichotomized into two pertinent categories $(1=17-18,2=19$ and older $)$. See Table 1 for a summary of the study variables.

\section{Gender (Time 1)}

Gender was self-reported and dummy-coded $(1=$ female, 2 = male $)$.

\section{Main study variables}

High school grade (Time 1)

HSG was self-reported and used as a proxy for actual performance in high school based on the strong relationship between the two, $r=.84$ (Perry, Hladkyj, Pekrun, Clifton, \& Chipperfield, 2005; $1=50 \%$ or less, $10=91-100 \%$ ). Previous research demonstrates this self-report measure of HSG is a strong and reliable predictor of achievement in university, including course grades, $r=.40-.54$; and overall GPAs, $r=.51-.54$ (e.g. Hamm, Perry, Clifton, Chipperfield, \& Boese, 2014; Perry et al., 2005, 2010; Perry, Hladkyj, Pekrun, \& Pelletier, 2001). 


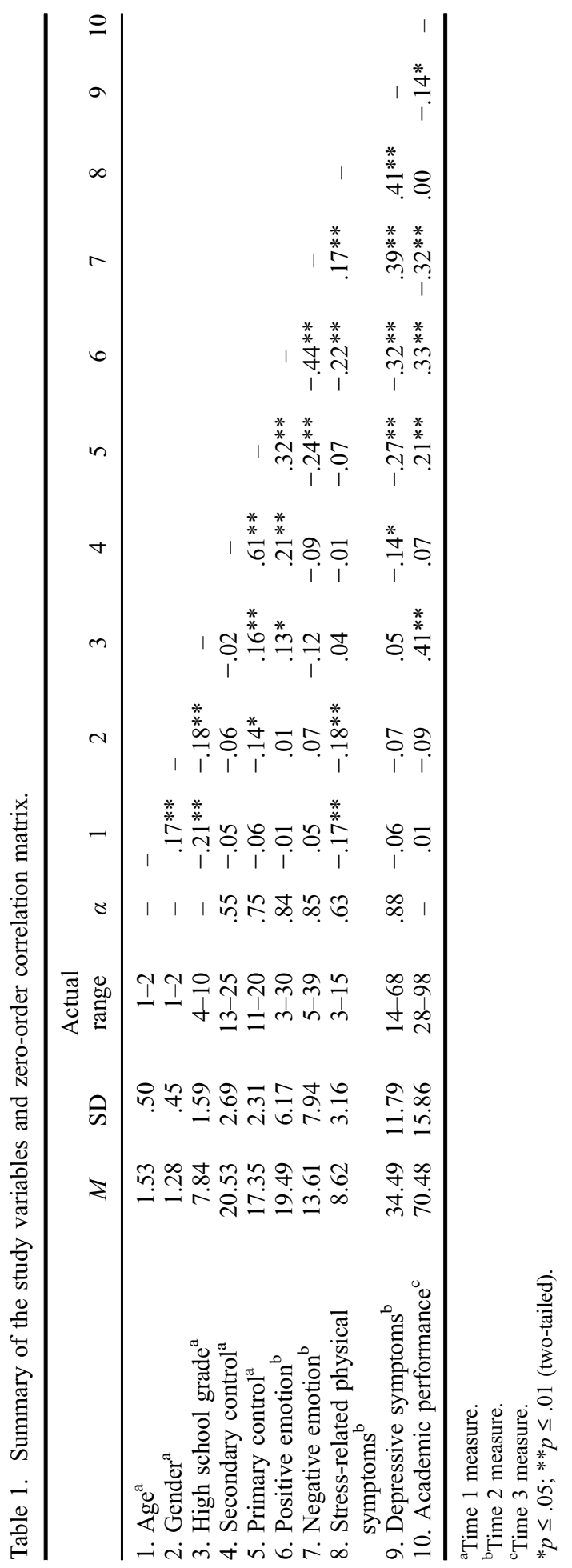


Selective secondary control strategies (Time 1)

Five items from the Academic-Specific Control Strategies (ASCS) scale were used to measure participants' selective secondary control striving (Hamm et al., 2013; 1 = strongly disagree, 5 = strongly agree; e.g. 'I often remind myself how important it is for my future to have a good education').

\section{Selective primary control strategies (Time 1)}

Four items from the ASCS scale were used to measure participants' selective primary control striving (e.g. 'I will put time and effort into my education whenever I can').

\section{Positive emotion (Time 2)}

Participants reported their happiness, pride and hope after reading the following stem: 'Rate the extent to which each of the following emotions describe how you feel about your performance in your introductory psychology course so far this year'. Ratings were provided on a 10 -point scale $(1=$ not at all, $10=$ very much so $)$.

\section{Negative emotion (Time 2)}

Participants reported their guilt, regret, helplessness, shame and anger after reading the same stem as that described for the positive emotions. Ratings were provided on the same 10-point scale.

\section{Depressive symptoms (Time 2)}

Participants reported the extent to which they experienced 14 depressive symptoms from Goldberg's Depression Inventory (Goldberg, 1993; 1 = not at all, $6=$ very much; e.g. 'I have lost interest in aspects of life that used to be important to me').

\section{Stress-related physical symptoms (Time 2)}

Based on the Cohen-Hoberman Inventory of Physical Symptoms (Cohen \& Hoberman, 1983), we assessed three physical symptoms commonly implicated in stress: headaches, muscle tension and fatigue (APA, 2012). Participants rated the frequency each symptom was experienced within the past two months $(1=$ not at all, $5=5$ or more times a month $)$.

\section{Academic performance (Time 3)}

Consenting students' academic performance was measured using their Time 3 test grades (percentages) from an introductory psychology course. Data were collected from course instructors after the second semester concluded.

\section{Results}

\section{Rationale for analyses}

Our primary interest was in selective secondary control's indirect effects on long-term health for students with low HSGs via the hypothesised sequence of psychological 
mechanisms (see Figure 1 for the specified models). Consequently, our path analytic approach involved using AMOS (maximum-likelihood method) to calculate the omnibus effects of predictor variables and assess model fit using chi-square $\left(\chi^{2}\right)$, the comparison fit index (CFI) and the root mean square error of approximation (RMSEA) based on recommendations by Byrne (2010). Hayes's (2013) PROCESS macro for SPSS was subsequently employed to conduct tests of moderated mediation by probing Secondary Control $\times$ HSG interaction effects. This approach allowed us to examine whether secondary control indirectly benefited students with low HSGs (i.e. whether secondary control had conditional indirect effects).

Secondary control's conditional indirect effects were examined at low ( -1 SD) and high (+1 SD) levels of HSG. We tested the significance of our directional hypotheses concerning these conditional indirect effects using a bootstrap approach that employed 90\% bias corrected confidence intervals (CIs; Hayes, 2013; Preacher \& Hayes, 2008). Mediation was confirmed if zero fell outside the CI based on 5000 samples of the unstandardised beta weights. Indirect effects of the omnibus predictors were tested using the same approach. Note that we correlated the residuals between positive and negative emotions to account for interrelationships between these constructs in accordance with previous research (e.g. Daniels et al., 2009). All effects were adjusted for participant age and gender in order to control for the influence of these demographic factors on our outcome measures.

\section{Preliminary analyses \\ Zero-order correlations}

Correlation coefficients revealed a number of logically consistent associations between the study variables (see Table 1). For instance, depressive symptoms exhibited a moderate and positive relationship with stress-related physical symptoms. Further, positive and negative emotion were related to each other and all dependent variables in expected directions. Students who frequently employed secondary control strategies reported higher levels of primary control and positive emotion, but fewer depressive symptoms. Although secondary control was not significantly related to negative emotion or stressrelated physical symptoms, this was consistent with our model which specified that secondary control's effects would be moderated by students' HSGs (i.e. primarily benefit those with low HSGs). In addition to its strong relationship with secondary control, primary control was related to positive emotion, negative emotion, depressive symptoms and academic performance in expected directions. Finally, HSG was the strongest correlate of performance, with positive emotion, negative emotion and depressive symptoms also significantly correlated.

\section{Simple effects of secondary control on health}

Prior to conducting the main analyses, we examined simple Secondary Control $\times$ HSG regression models to determine whether secondary control benefited our health outcomes for students with low HSGs in the absence of the mediators. The Secondary Control $\times$ HSG interactions were significant for both depressive $(\beta=.17, p=.009)$ and stress-related physical $(\beta=.17, p=.009)$ symptoms despite controlling for age and 
gender. More interestingly, simple slope analyses suggested that secondary control predicted fewer depressive symptoms for those with low $(\beta=-.33, p=.001)$ but not high HSGs $(\beta=.01, p=.873)$. Similarly, secondary control predicted fewer stress-related physical symptoms for those with low $(\beta=-.20, p=.031)$ but not high HSGs $(\beta=.14$, $p=.120)$. Thus, these analyses provide preliminary support for our hypotheses and suggest that secondary control sustains health for students facing challenging educational obstacles. The following path analyses serve to further explicate our model by examining theorized mechanisms that may account for secondary control's effects.

\section{Main analyses}

\section{Model fit summary}

We specified and tested separate structural models predicting depressive symptoms and stress-related physical symptoms (see Figure 1). Results from these two path models indicated that the models fit the data well: depressive symptoms model, $\chi^{2}(6)=6.79$, $p=.340 ; \mathrm{CFI}=.997 ; \mathrm{RMSEA}=.024$; stress-related physical symptoms model, $\chi^{2}(6)$ $=9.37, p=.154 ; \mathrm{CFI}=.986 ; \mathrm{RMSEA}=.049$. However, the individual path estimates in each model provided direct tests of our hypotheses and were therefore of greatest interest. Path estimates are detailed separately for each model below.

\section{Depressive symptoms model}

The individual path estimates supported our predictions (see Figure 2) and demonstrated that the Secondary Control $\times$ HSG interaction was a significant predictor of primary control $(\beta=-.17, p<.001)$. Simple slope analyses revealed that the positive influence of secondary control on primary control was nearly twice as strong when HSG was low $(\beta=.79, p<.001)$ than when HSG was high $(\beta=.44, p<.001$; see Figure 3$){ }^{4}$

In line with predictions, primary control reliably predicted positive $(\beta=.32$, $p<.001)$ and negative emotion $(\beta=-.23, p<.001)$. Because secondary control predicted primary control, which in turn predicted the emotions, we tested whether secondary control had conditional indirect effects on the emotions through primary control. As expected, when HSG was low, secondary control had a strong indirect influence on positive $(\beta=.25, \mathrm{CIs}=.2333$ to .7195$)$ and negative $(\beta=-.18, \mathrm{CIs}=-.9044$ to -.2842 ) emotion via primary control. When HSG was high, secondary control's influence on positive $(\beta=.14$, CIs $=.1293$ to .4464$)$ and negative $(\beta=-.10$, CIs $=-.5568$ to -.1607$)$ emotion remained significant but was notably reduced.

Consistent with our hypotheses, primary control $(\beta=-.18, p=.003)$, positive emotion $(\beta=-.15, p=.027)$ and negative emotion $(\beta=.31, p<.001)$ predicted depressive symptoms. As expected, tests of mediation demonstrated that primary control also indirectly predicted depressive symptoms via its influence on positive $(\beta=-.05$, CIs $=-.4845$ to -.0471$)$ and negative emotion $(\beta=-.07$, CIs $=-.6152$ to -.1781$){ }^{5}$

\section{Stress-related physical symptoms model}

Note that the only distinction between our depressive symptoms and stress-related physical symptoms models was that they predicted different outcomes (i.e. we employed the same model to predict both outcomes; see Figure 1). As a consequence, 


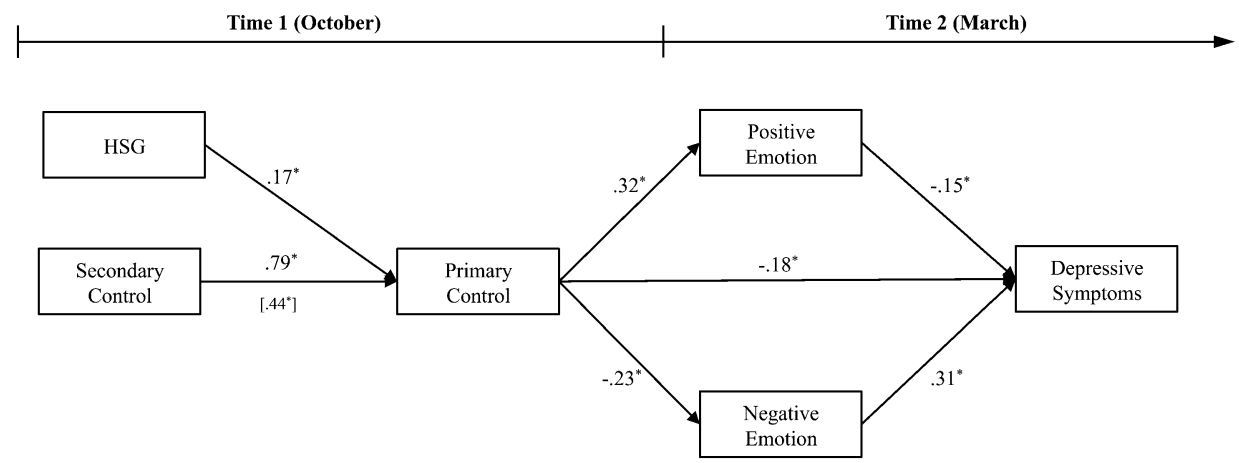

Figure 2. Conditional indirect effects of secondary control on depressive symptoms. Standardised regression weights are reported for all effects.

Notes: To facilitate interpretation, the omnibus effects of secondary control and the Secondary Control $\times$ HSG interaction term are not shown. Rather, the paths from secondary control to primary control are presented separately for low ( -1 SD) and high (+1 SD) HSG: low HSG is reported above the arrow, and high HSG is reported below the arrow [in brackets]. All effects control for age and gender. Residuals and non-significant paths are not shown. ${ }^{*} p \leq .05$ (twotailed).

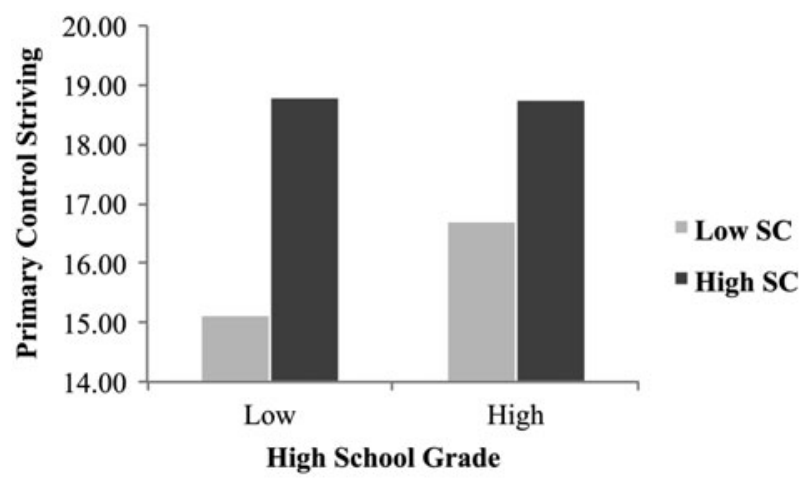

Figure 3. Conditional effects of secondary control (SC) on primary control striving at low $(-1$ $\mathrm{SD})$ and high (+1 SD) levels of HSG.

all path estimates in the present model were identical to those reported above with the exception of those relating to stress-related physical symptoms. Thus, we report only path estimates involving the prediction of stress-related physical symptoms below.

Individual path estimates revealed that positive emotion $(\beta=-.16, p=.025)$ predicted stress-related physical symptoms. Negative $(\beta=.11, p=.099)$ emotion was a marginally significant predictor of these symptoms. Although primary control did not directly predict stress-related physical symptoms, tests of mediation demonstrated that primary control indirectly predicted this health outcome via its influence on positive $(\beta=-.05$, CIs $=-.1371$ to -.0157$)$ and negative emotion $(\beta=-.03$, CIs $=-.0833$ to -.0015$)$. 


\section{Supplemental analyses: Secondary control and goal attainment}

Our supplemental analyses explored whether secondary control had indirect effects on Time 3 academic performance for students with low HSGs via the hypothesised sequence of psychological mechanisms. Once again, with the exception of path estimates relating to performance, all effects were identical to those reported above. Thus, we report only the path estimates involving the prediction of academic performance below. ${ }^{6}$

Results from the academic performance path analysis suggested our model fit the data well: $\chi^{2}(6)=7.532, p=.274$; CFI $=.995$; RMSEA $=.033$. More interesting, individual path estimates revealed that positive $(\beta=.18, p=.004)$ and negative $(\beta=-.18$, $p=.003)$ emotion predicted Time 3 academic performance. Unsurprisingly, HSG $(\beta=.37, p<.001)$ was also a strong predictor of student performance. Although primary control did not directly predict performance, tests of mediation demonstrated that primary control indirectly predicted this outcome via its influence on positive $(\beta=.06$, $\mathrm{CIs}=.1557$ to .7738$)$ and negative emotion $(\beta=.04$, CIs $=.1195$ to .5284$)$. Thus, consistent with the main analyses, the supplemental analyses suggested secondary control indirectly influenced goal attainment for those with low HSGs via the hypothesised sequence of psychological mechanisms. ${ }^{7}$

\section{Discussion}

Although health is commonly believed to flourish in young adulthood, it may suffer among those navigating the challenging developmental transition from high school to university. Young adults report elevated levels of stress and are at increased risk of depression relative to those in the general population (APA, 2012; ACHA, 2012; Kessler et al., 2005). The deleterious effects of this transition may be even more pronounced for those who are unprepared and face the additional obstacle of having to overcome a history of poor academic performance. Supporting this rationale, standardised predicted values $\left(Z_{\text {PRED }}\right)$ based on the preliminary analyses are provided in parentheses below and highlight the plight of students with low HSGs (-1 SD) who infrequently employed selective secondary control strategies $(-1 \mathrm{SD})$ : These individuals experienced the most stress-related physical symptoms $\left(Z_{\text {PRED }}=.22\right)$, the most depressive symptoms $\left(Z_{\text {PRED }}=.28\right)$, and the lowest academic performance $\left(Z_{\text {PRED }}=-.66\right)$.

In contrast, their peers with low HSGs $(-1 \mathrm{SD})$ who frequently employed selective secondary control strategies $(+1 \mathrm{SD})$ experienced considerably lower levels of stress-related physical $\left(Z_{\mathrm{PRED}}=-.15\right)$ and depressive $\left(\mathrm{Z}_{\mathrm{PRED}}=-.31\right)$ symptoms, and performed notably better on their Time 3 test $\left(Z_{\mathrm{PRED}}=-.21\right)$. Moreover, these predicted values for students with low HSGs and high selective secondary control were largely in line with their notat-risk peers who entered university with high HSGs. Thus, our results indicate that students with low HSGs may be especially vulnerable to the harmful effects of the school-to-university transition. Encouragingly, however, our findings also suggest that motivation-focused thinking may protect against these detriments for at-risk individuals with low HSGs.

\section{Selective secondary control's conditional indirect effects on health and performance}

Our results and the preceding discussion indicate the value of selective secondary control for young adults facing a major personal barrier to goal attainment: entering 
university with a low HSG. As hypothesised, and consistent with the motivational theory of lifespan development (Heckhausen et al., 2010), these findings suggest that selective secondary control is most needed (and most beneficial) when individuals face significant challenges during goal pursuit. And yet, although we were invested in determining which individuals benefit most from these motivation-focused strategies, we were equally concerned with elucidating the mechanisms through which selective secondary control exerted its influence. In particular, we predicted that the advantages of selective secondary control would be indirect through primary control and previously unexamined measures of emotion.

In accordance with our hypotheses, simple slope analyses demonstrated that selective secondary control was nearly twice as strong a predictor of selective primary control when HSG was low $(\beta=.79)$ than when HSG was high $(\beta=.44)$. Further, primarily for students with low HSGs, selective secondary control indirectly predicted five-month measures of positive and negative emotion via selective primary control. Particularly noteworthy is a comparison of the magnitudes of selective secondary control's indirect effects for students with low vs. high HSGs. For students with low HSGs, a $1 \mathrm{SD}$ increase in selective secondary control corresponded to a $.25 \mathrm{SD}$ increase in positive emotion and a .18 SD decrease in negative emotion through selective primary control. In contrast, for students with high HSGs, a 1 SD increase in selective secondary control corresponded to notably smaller changes in positive (.14 SD) and negative $(.10 \mathrm{SD})$ emotion through selective primary control.

The latter half of our models suggests there may be long-term implications to selective secondary control's influence on selective primary control and the emotions, as these mediators provided mechanisms through which motivation-focused thinking sustained health. Among students with low HSGs, selective secondary control was related to reduced depressive symptoms via the following paths: selective primary control $(\beta=-.14)$, selective primary control-positive emotion $(\beta=-.04)$ and selective primary control-negative emotion $(\beta=-.05)$. Thus, a 1 SD increase in selective secondary control corresponded to approximately a $.25 \mathrm{SD}$ decrease in depressive symptoms assessed five months later via the proposed mediators in the hypothesised causal sequence. ${ }^{8}$

This pattern was replicated for stress-related physical symptoms. When HSG was low, selective secondary control predicted fewer of these symptoms through the selective primary control-positive emotion $(\beta=-.04)$ and selective primary control-negative emotion $(\beta=-.02)$ paths. Thus, our depression model accounted for a sizable $70 \%$ of selective secondary control's effect on depressive symptoms given that the preliminary analyses indicated the influence of these motivation-focused strategies was $\beta=-.33$ in the absence of the mediators. Our stress-related physical symptom model also accounted for $30 \%$ of selective secondary control's effect on these symptoms when considering the preliminary analyses demonstrated the influence of selective secondary control was $\beta=-.20$ in the absence of the mediators.

The supplementary analyses indicate that selective secondary control's conditional indirect effects during a challenging developmental transition are not limited to the health domain. For students with low HSGs, selective secondary control strategies indirectly promoted sixth-month academic performance through the selective primary control-positive emotion $(\beta=.05)$ and selective primary control-negative emotion paths $(\beta=.03)$. Highlighting low HSG as a significant impediment to post-secondary achievement, our study is in line with the broader empirical literature in documenting HSG's 
strong relationship with university course grades ( $r=.40-.54$; Perry et al., 2001, 2010). Encouragingly, however, our findings suggest that this barrier may be partly overcome by employing selective secondary control strategies.

Collectively, our results indicate that, particularly for young adults facing a major educational obstacle, selective secondary control indirectly promotes health and performance through increased selective primary control striving and emotional well-being. Thus, our study represents the first simultaneous test of two interconnected theoretical propositions proposed by Heckhausen et al. (2010) that have previously been explored only in isolation (Hamm et al., 2013; Poulin \& Heckhausen, 2007): that the benefits of selective secondary control should be indirect and primarily advantage those encountering barriers during goal pursuit. Moreover, our study expands on previous theory and research by demonstrating that selective secondary control exerts its influence, in part, by positively impacting discrete emotions among young adults. Hence, selective secondary control may help young adults in transition overcome challenging obstacles by indirectly increasing positive emotion and reducing negative emotion via selective primary control. Finally, our study focused on selective secondary control's effects on critical physical and psychological health outcomes for young adults that have been neglected in past studies of the construct, namely stress-related physical symptoms and depressive symptomology.

Our results also have implications for university students navigating the challenging transition from high school to university. As documented earlier, young adults enrolled in post-secondary education must cope with psychological and physical health problems that are equivalent to, or potentially even more severe than, the general population (ACHA, 2012; Adlaf et al., 2001; Grace, 1997; Hussain et al., 2013). Our study suggests that, for students facing the added challenge of entering university with a low HSG, selective secondary control striving may act as a buffer that reduces the risk of experiencing elevated stress-related physical symptoms and depression. Given the strong relationship between depression and suicide, and the elevated risk of suicide in young adulthood (Galaif, Sussman, Newcomb, \& Locke, 2007; Westefeld et al., 2005), employing selective secondary control strategies may have critical long-term consequences for university students facing challenging obstacles.

\section{Strengths, limitations and future directions}

The present study is supported by the use of two critical measures of health for young adults, a six-month design, and three measurement points involving a combination of psychological, physical and performance measures. Further, our research was based on the motivational theory of lifespan development (Heckhausen et al., 2010), which has amassed impressive empirical support for its core tenets, but has inspired few studies on the potential health benefits of selective secondary control striving for young adults. Hence, the present study supplements the existing literature by using path analysis and moderated mediation to document not only the long-term health consequences of selective secondary control, but also to provide insight into which individuals are most advantaged by these motivation-focused strategies and the mechanisms through which these benefits are realised.

Our study has several limitations. First, although our models predicting depressive and stress-related physical symptoms imply four separate steps, data on these measures 
were collected at only two time points. Hence, the secondary control-primary control and emotion-depressive/physical symptom paths were based on cross-sectional data from Time 1 and Time 2, respectively. However, the supplemental autoregressive analyses reported in Notes 4, 5 and 7 substantiate our main results and indicate that all significant paths in the hypothesised model remain reliable when accounting for pre-existing differences in the dependent variables. Second, our measure of HSG was self-reported and may therefore not perfectly reflect actual high school achievement. However, previous research indicates this self-reported measure of HSG is strongly related to actual HSGs, $r=.84$ (Perry et al., 2005). Further, to the extent that the barriers to university achievement associated with low HSGs are due to beliefs about personal aptitude, self-reported HSG may better reflect the type of everyday obstacle we intended to assess than would actual HSG. Third, although our outcome measures were relatively broad indicators of stress-related physical and depressive symptoms, our emotion measures were limited to the course-specific indicators available in the present cohort. However, the results may have been stronger if broader indicators of emotion were used since such measures would better match our broad outcome measures.

Although our study sheds further light on selective secondary control, many questions about the construct remain. For instance, are there circumstances under which engaging in selective secondary control strategies are actually maladaptive? Results observed for students with high HSGs in the preliminary analyses imply that such conditions may exist: For those with high HSGs, selective secondary control was related to marginally higher reports of stress-related physical symptoms $(\beta=.14$, $p=.120$ ). Given that selective secondary control is posited to primarily benefit those facing challenging circumstances (Heckhausen et al., 2010), it may be that employing these strategies when facing few obstacles is not only unnecessary, but harmful. Supporting this rationale, recent research points to one instance under which selective secondary control can be detrimental: Among very old adults, there are negative health consequences to engaging in selective secondary control strategies when corresponding selective primary control strategies are not employed (Hamm, Chipperfield et al., 2014). Thus, future research would do well to further explore this issue.

An additional avenue for future research involves examining alternative mechanisms that may transmit selective secondary control's effects. In particular, Heckhausen et al. (2010) theorize that these strategies should also enhance motivational commitment to important goals. Consequently, there would be theoretical and practical value to investigating whether selective secondary control enhances the value of chosen goals (e.g. physical fitness) and diminishes the value of competing goals (e.g. leisure time), particularly among those who face challenging obstacles (e.g. individuals who are overweight). Finally, our results have implications for the development of treatment interventions. Preliminary evidence suggests that a treatment designed to promote the use of motivation-focused selective secondary control strategies may enhance long-term emotional well-being and academic performance among young adults facing challenging circumstances (Chipperfield et al., 2013). However, further research is needed to replicate and extend these findings to determine whether the treatment impacts critical measures of psychological and physical health. 


\section{Conclusion}

Although the transition from high school to university is commonly conceived of as a period of opportunity, this shift is imbued with uncertainty and unpredictability which can undermine health (Adlaf et al., 2001; Hussain et al., 2013; Perry, 2003). Our study builds on previous research by exploring whether selective secondary control striving facilitates health and performance among young adults facing major educational obstacles. Results suggest that, for students with low HSGs, selective secondary control indirectly reduces long-term stress-related physical and depressive symptoms (and promotes academic performance) through selective primary control and previously unexamined measures of discrete emotions. Thus, by employing these motivation-focused strategies, young adults facing difficult obstacles may be able to enhance their goal striving and emotional well-being, and thereby sustain their psychological and physical health.

\section{Disclosure statement}

No potential conflict of interest was reported by the authors.

\section{Funding}

This work was supported by a Social Sciences and Humanities Research Council of Canada (SSHRC) Doctoral Fellowship and a Manitoba Graduate Scholarship to the first author, a SSHRC insight grant [435-2012-1143] and a Royal Society of Canada and Alexander von Humboldt research grant to the second author, and an operating grant from SSHRC [410-2010-2049] to the third author.

\section{Notes}

1. This calculation is based on an $11 \%$ prevalence among American university students (ACHA, 2012) relative to a 7\% prevalence in the American general population (Kessler et al., 2005).

2. Note that although the transition from high school to university can be construed as a discrete shift (e.g. high school students become university students on the first day of class), we regard the process of adapting to this major developmental transition as one that occurs over an extended period of time and throughout the first year of university. This is because many first-year students struggle to successfully navigate novel, unpredictable and ongoing challenges during this transition that include new living arrangements, financial responsibilities, critical career choices, novel learning environments, increased pressures to excel and frequent failures (see Perry, 1991, 2003; Perry, Stupnisky, Hall, Chipperfield, \& Weiner, 2010).

3. Note that in densely populated regions containing a variety of academic institutions, some students with low HSGs may calibrate their level of challenge by choosing to enrol in less challenging universities and programmes. However, this is unlikely to be the case for low HSG students in the present sample who were enrolled at the only large, research-intensive university in an expansive Canadian province with a small population and who therefore had limited options for post-secondary education. More broadly, evidence suggests many university students may struggle to calibrate their level of challenge given that national estimates indicate nearly $30 \%$ of freshman withdraw from their institutions and less than $60 \%$ graduate after six years (Snyder \& Dillow, 2013).

4. Secondary $\left(r_{T 1-T 2}=.61\right)$ and primary $\left(r_{T 1-T 2}=.63\right)$ control were also assessed at Time 2 , and both measures exhibited moderate stability over time. Assessing primary control at Time 2 also enabled us to examine whether secondary control's effects remained reliable when accounting for autoregressive effects. Simple slope analyses indicated secondary control 
remained a significant predictor of Time 2 primary control at low and high HSG despite accounting for variability in the Time 1 measure.

5. Because data on Time 2 measures were also collected at Time 1 (i.e. positive and negative emotion, depressive symptoms and stress-related physical symptoms), we conducted supplemental ordinary least square regression (OLS) analyses to determine whether significant paths in the depressive and stress-related physical symptoms models remained reliable when accounting for autoregressive effects. All effects remained significant when accounting for variability in the Time 1 measures.

6. As part of our supplemental analyses, we conducted a simple Secondary Control $\times$ HSG moderation model to determine whether secondary control promoted performance for students with low HSGs in the absence of the mediators. Consistent with the preliminary results reported earlier, these simple slope analyses revealed that secondary control predicted increased performance for those with low $(\beta=.24, p=.007)$ but not high $(\beta=-.06$, $p=.427)$ HSGs despite controlling for age and gender.

7. Data on students' first test scores (September) were also collected, and supplemental OLS regression analyses indicated that both positive and negative emotion remained reliable predictors of Time 3 test performance (April) when accounting for autoregressive effects (i.e. students' initial test scores).

8. Calculations of the magnitudes of selective secondary control's indirect effects on the health/ performance outcomes are based on only significant individual paths.

\section{References}

Adlaf, E. M., Gliksman, L., Demers, A., \& Newton-Taylor, B. (2001). The prevalence of elevated psychological distress among Canadian undergraduates: Findings from the 1998 Canadian Campus Survey. Journal of American College Health, 50, 67-72. doi:10.1080/ 07448480109596009

American College Health Association. (2012). American College Health Association national college health assessment II: Reference group executive summary fall 2012. Hanover, MD.

American Psychological Association. (2012). Stress in America: Our health at risk. Retrieved from http://www.apa.org/news/press/releases/stress/2011/final-2011.pdf

Byrne, B. M. (2010). Structural equation modeling with AMOS: Basic concepts, applications, and programming (2nd ed.). New York, NY: Routledge, Taylor \& Francis Group.

Chipperfield, J. G., Hamm, J. M., Perry, R. P., Hladkyj, S., Parker, P. C., Rodriguez, P., \& Dubberley, K. (2013, January). An intervention to enhance learning-related emotions and performance in college students. Hawaiian International Conference on Education. Honolulu, Hawaii.

Chipperfield, J. G., \& Perry, R. P. (2006). Primary- and secondary-control strategies in later life: Predicting hospital outcomes in men and women. Health Psychology, 25, 226-236. doi:10.1037/0278-6133.25.2.226

Chipperfield, J. G., Perry, R. P., \& Menec, V. H. (1999). Primary and secondary control-enhancing strategies: Implications for health in later life. Journal of Aging and Health, 11, 517-539. doi: $10.1177 / 089826439901100403$

Chipperfield, J. G., Perry, R. P., \& Weiner, B. (2003). Discrete emotions in later life. The Journals of Gerontology Series B: Psychological Sciences and Social Sciences, 58, 23-34. doi:10.1093/geronb/58.1.P23

Cohen, S., \& Hoberman, H. (1983). Positive events and social supports as buffers of life change stress. Journal of Applied Social Psychology, 13, 99-125. doi:10.1111/j.15591816.1983.tb02325.x

Daniels, L. M., Stupnisky, R. H., Pekrun, R., Haynes, T. L., Perry, R. P., \& Newall, N. E. (2009). A longitudinal analysis of achievement goals: From affective antecedents to emotional effects 
and achievement outcomes. Journal of Educational Psychology, 101, 948-963. doi:10.1037/ a0016096

Galaif, E. R., Sussman, S., Newcomb, M. D., \& Locke, T. F. (2007). Suicidality, depression, and alcohol use among adolescents: A review of empirical findings. International Journal of Adolescent Medical Health, 19, 27-35. doi:10.1515/IJAMH.2007.19.1.27

Goldberg, I. K. (1993). Questions and answers about depression and its treatment: Consultation with a leading psychiatrist. Philadelphia, PA: Charles Press.

Grace, T. W. (1997). Health problems of college students. Journal of American College Health, 45, 243-251. doi:10.1080/07448481.1997.9936894

Haase, C. M., Heckhausen, J., \& Köller, O. (2008). Goal engagement during the school-work transition: Beneficial for all, particularly for girls. Journal of Research on Adolescence, 18, 671-698. doi:10.1111/j.1532-7795.2008.00576.x

Haase, C. M., Heckhausen, J., \& Silbereisen, R. K. (2012). The interplay of occupational motivation and well-being during the transition from university to work. Developmental Psychology, 48, 1739-1751. doi:10.1037/a0026641

Hall, N. C., Chipperfield, J. G., Heckhausen, J., \& Perry, R. P. (2010). Control striving in older adults with serious health problems: A 9-year longitudinal study of survival, health, and wellbeing. Psychology and Aging, 25, 432-445. doi:10.1037/a0019278

Hamm, J. M., Chipperfield, J. G., Perry, R. P., Heckhausen, J., \& Mackenzie, C. S. (2014). Conflicted goal engagement: Undermining physical activity and health in late life. The Journals of Gerontology Series B: Psychological Sciences and Social Sciences, 69, 533-542. doi:10.1093/geronb/gbu048

Hamm, J. M., Perry, R. P., Clifton, R. A., Chipperfield, J. G., \& Boese, G. D. (2014). Attributional retraining: A motivation treatment with differential psychosocial and performance benefits for failure prone individuals in competitive achievement settings. Basic and Applied Social Psychology, 36, 221-237. doi:10.1080/01973533.2014.890623

Hamm, J. M., Stewart, T. L., Perry, R. P., Clifton, R. A., Chipperfield, J. G., \& Heckhausen, J. (2013). Sustaining primary control striving for achievement goals during challenging developmental transitions: The role of secondary control strategies. Basic and Applied Social Psychology, 35, 286-297. doi:10.1080/01973533.2013.785404

Hayes, A. F. (2013). Introduction to mediation, moderation, and conditional process analysis: A regression-based approach. New York, NY: The Guilford Press.

Haynes, T. L., Heckhausen, J., Chipperfield, J. G., Perry, R. P., \& Newall, N. E. (2009). Primary and secondary control strategies: Implications for health and well-being among older adults. Journal of Social and Clinical Psychology, 28, 165-197. doi:10.1521/jscp.2009.28.2.165

Heckhausen, J., \& Schulz, R. (1995). A life-span theory of control. Psychological Review, 102, 284-304. doi:10.1037/0033-295X.102.2.284

Heckhausen, J., Wrosch, C., \& Fleeson, W. (2001). Developmental regulation before and after a developmental deadline: The sample case of "biological clock" for childbearing. Psychology and Aging, 16, 400-413. doi:10.1037/0882-7974.16.3.400

Heckhausen, J., Wrosch, C., \& Schulz, R. (2010). A motivational theory of life-span development. Psychological Review, 117, 32-60. doi:10.1037/a0017668

Hussain, R., Guppy, M., Robertson, S., \& Temple, E. (2013). Physical and mental health perspectives of first year undergraduate rural university students. BMC Public Health, 13(848), 1-11.

Kessler, R. C., Chiu, W. T., Demler, O., \& Walters, E. E. (2005). Prevalence, severity, and comorbidity of 12-month DSM-IV disorders in the national comorbidity survey replication. Archives of General Psychiatry, 62, 617-627. doi:10.1001/archpsyc.62.6.617

Lyubomirsky, S., King, L., \& Diener, E. (2005). The benefits of frequent positive affect: Does happiness lead to success? Psychological Bulletin, 131, 803-855. doi:10.1037/00332909.131.6.803 
Mackay, J., Charles, S. T., Kemp, B., \& Heckhausen, J. (2011). Goal striving and maladaptive coping in adults living with spinal cord injury: Associations with affective well-being. Journal of Aging and Health, 23, 158-176. doi:10.1177/0898264310382039

Ostir, G., Markides, K. S., Black, S. A., \& Goodwin, J. S. (2000). Emotional well-being predicts subsequent functional independence and survival. Journal of the American Geriatrics Society, $48,473-478$.

Pekrun, R., Goetz, T., Perry, R. P., Kramer, K., Hochstadt, M., \& Molfenter, S. (2004). Beyond test anxiety: Development and validation of the test emotions questionnaire (TEQ). Anxiety, Stress, and Coping, 17, 287-316. doi:10.1080/10615800412331303847

Perry, R. P. (1991). Perceived control in college students: Implications for instruction in higher education. In J. Smart (Ed.), Higher education: Handbook of theory and research (Vol. 7, pp. 1-56). New York, NY: Agathon Press.

Perry, R. P. (2003). Perceived (academic) control and causal thinking in achievement settings. Canadian Psychology/Psychologie Canadienne, 44, 312-331. doi:10.1037/h0086956

Perry, R. P., Hladkyj, S., Pekrun, R. H., Clifton, R. A., \& Chipperfield, J. G. (2005). Perceived academic control and failure in college students: A three-year study of scholastic attainment. Research in Higher Education, 46, 535-569. doi:10.1007/s11162-005-3364-4

Perry, R. P., Hladkyj, S., Pekrun, R. H., \& Pelletier, S. T. (2001). Academic control and action control in the achievement of college students: A longitudinal field study. Journal of Educational Psychology, 93, 776-789. doi:10.1037/0022-0663.93.4.776

Perry, R. P., Stupnisky, R. H., Hall, N. C., Chipperfield, J. G., \& Weiner, B. (2010). Bad starts and better finishes: Attributional retraining and initial performance in competitive achievement settings. Journal of Social and Clinical Psychology, 29, 668-700. doi:10.1521/ jscp.2010.29.6.668

Poulin, M. J., \& Heckhausen, J. (2007). Stressful events compromise control strivings during a major life transition. Motivation and Emotion, 31, 300-311. doi:10.1007/s11031-007-9077-6

Preacher, K. J., \& Hayes, A. F. (2008). Asymptotic and resampling strategies for assessing and comparing indirect effects in multiple mediator models. Behavior Research Methods, 40, 879-891. doi:10.3758/BRM.40.3.879

Richardson, M., Abraham, C., \& Bond, R. (2012). Psychological correlates of university students' academic performance: A systematic review and meta-analysis. Psychological Bulletin, 138, 353-387. doi:10.1037/a0026838

Schilling, O. K., Wahl, H. W., Boerner, K., Reinhardt, J. P., Brennan-Ing, M., \& Horowitz, A. (2013). Change in psychological control in visually impaired older adults over 2 years: Role of functional ability and depressed mood. The Journals of Gerontology Series B: Psychological Sciences and Social Sciences, 68, 750-761. doi:10.1093/geronb/gbs118

Snyder, T. D., \& Dillow, S. A. (2013). Digest of Education Statistics 2012 (NCES 2014-015). Washington, DC: National Center for Education Statistics, Institute of Education Sciences, U.S. Department of Education.

Strenze, T. (2007). Intelligence and socioeconomic success: A meta-analytic review of longitudinal research. Intelligence, 35, 401-426. doi:10.1016/j.intell.2006.09.004

Wahl, H., Becker, S., Burmedi, D., \& Schilling, O. (2004). The role of primary and secondary control in adaptation to age-related vision loss: A study of older adults with macular degeneration. Psychology and Aging, 19, 235-239. doi:10.1037/0882-7974.19.1.235

Westefeld, J. S., Homaifar, B., Spotts, J., Furr, S., Range, L., \& Werth, Jr. J. L. (2005). Perceptions concerning college student suicide: Data from four universities. Suicide and LifeThreatening Behavior, 35, 640-645. doi:10.1521/suli.2005.35.6.640

Windsor, T. D. (2009). Persistence in goal striving and positive reappraisal as psychosocial resources for ageing well: A dyadic analysis. Aging and Mental Health, 13, 874-884. doi:10.1080/13607860902918199 
Wrosch, C., \& Schulz, R. (2008). Health-engagement control strategies and 2-year changes in older adults' physical health. Psychological Science, 19, 537-541. doi:10.1111/j.14679280.2008.02120.x

Wrosch, C., Schulz, R., Miller, G. E., Lupien, S., \& Dunne, E. (2007). Physical health problems, depressive mood, and cortisol secretion in old age: Buffer effects of health engagement control strategies. Health Psychology, 26, 341-349. doi:10.1037/0278-6133.26.3.341 